\section{Conformable metallic stent placement in a sigmoid volvulus: a feasible and effective bridge option}

Sigmoid volvulus is the third leading cause of colonic obstruction in adults. Surgical resection with primary anastomosis is the standard of care [1]. Endoscopic reduction is a feasible option in patients with uncomplicated conditions [2] despite the fact that mechanical detorsion is not always successful, even by skilled hands. Few data are available concerning the role of self-expanding metallic stents (SEMS) in the treatment of benign colorectal strictures, including sigmoid volvulus.

An 82-year-old man was admitted because of intestinal occlusion. Full blood count and chemistry were normal but the computed tomography (CT) scan showed a sigmoid volvulus ( $\bullet$ Fig.1a). Following consultation with the surgeon, an attempt was made to reduce the volvulus endoscopically in order to schedule elective surgery. Unfortunately, the attempt at detorsion failed, so instead a Niti-S enteral uncovered stent (D-Type; TaeWoong, Seoul, Korea) was placed, as shown in - Fig. 1 b. The patient's symptoms improved rapidly, and 48 hours later he underwent resection of the volvulus. The patient was discharged in a good clinical condition after 7 days.

Nonresective alternatives have been used in sigmoid volvulus but with mixed results, and there are no large randomized controlled trials comparing stent placement with colonic resection [1]. It is widely accepted that SEMS placement for benign colonic obstruction due to diverticular stricture is not useful (high complication rates $[3,4])$, and very few data are available on the bridge-to-surgery efficacy of SEMS in treating twisted colon. The new Niti-S enteral uncovered stent (D-Type) is made of nitinol wire, which provides a flexible, fine mesh tubular prosthesis with eight radiopaque markers for an accurate release. Our experience suggests that this type of stent may be an effective and safe bridge option in patients with uncomplicated conditions who have sigmoid volvulus. This is because of the D-Type's conformability, which facilitates immediate, continuous wall apposition, and which in turn decompresses the colon until mandatory resective surgery can be performed.

\section{Endoscopy_UCTN_Code_TTT_1AQ_2AF}

\section{Competing interests: None}

\section{R. Di Mitri ${ }^{1}$, F. Mocciaro' ${ }^{1}$, S. Morini², M. C. Provenzano ${ }^{3}$, A. Marino}

${ }^{1}$ Gastroenterology and Endoscopy Unit, A.R.N.A.S. Civico Di Cristina-Benfratelli Hospital, Palermo, Italy

2 Emergency Surgery Unit, A.R.N.A.S.

Civico Di Cristina-Benfratelli Hospital, Palermo, Italy

${ }^{3}$ Radiology Unit, A.R.N.A.S. Civico Di Cristina-Benfratelli Hospital, Palermo, Italy
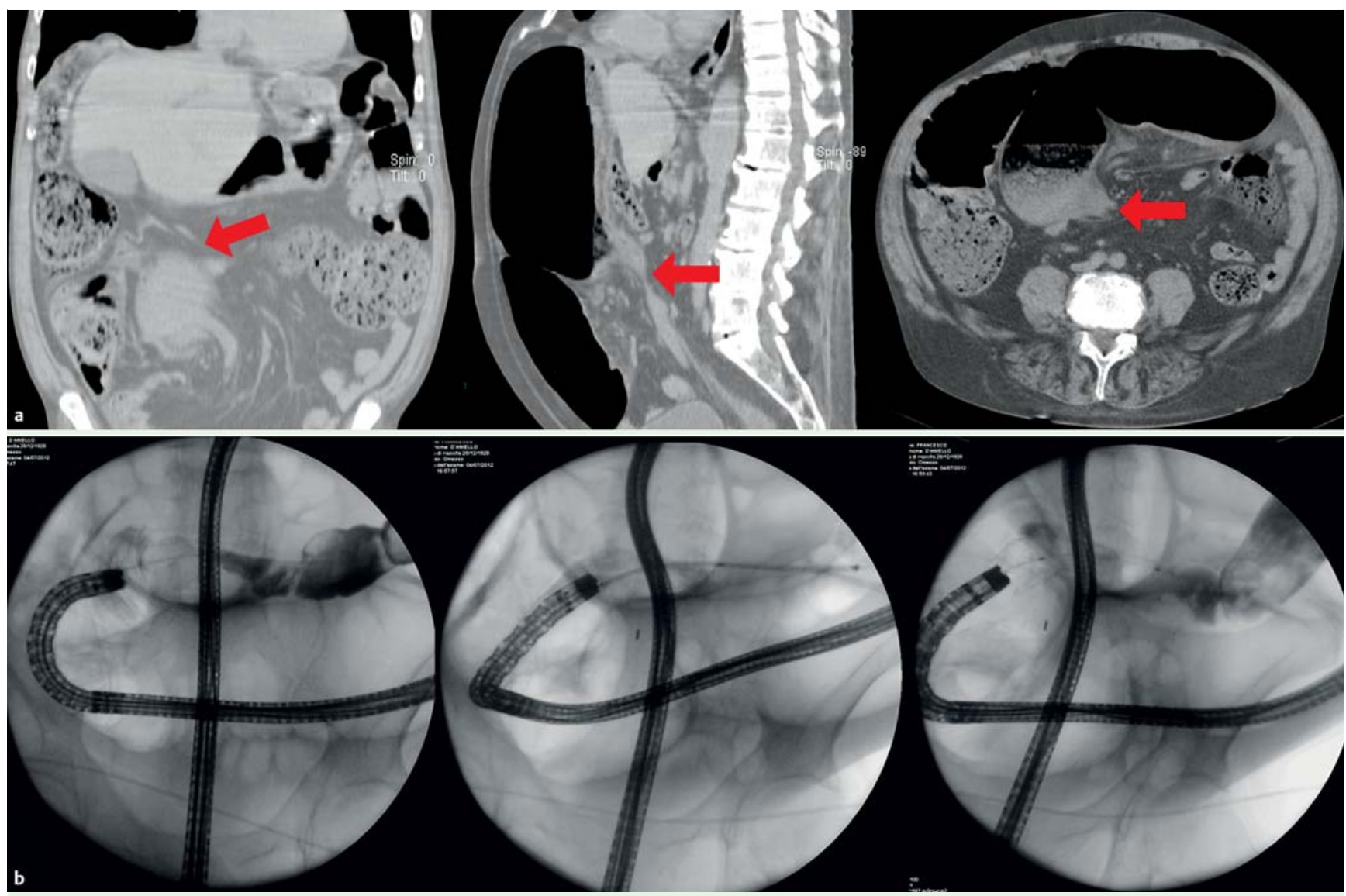

Fig. 1 Computed tomography scan of 82-year-old patient with intestinal occlusion. a Scan shows the sigmoid volvulus (red arrow). b Phases of the placement of a self-expanding metallic stent. 


\section{References}

1 Osiro SB, Cunningham D, Shoja MM et al. The twisted colon: a review of sigmoid volvulus. Am Surg 2012; 78: 271 - 279

2 Katsikogiannis N, Machairiotis N, Zarogoulidis $P$ et al. Management of sigmoid volvulus avoiding sigmoid resection. Case Rep Gastroenterol 2012; 6: 293-299

3 Small AJ, Young-Fadok TM, Baron TH. Expandable metal stent placement for benign colorectal obstruction: outcomes for 23 cases. Surg Endosc 2008; 22: 454-462
4 Forshaw MJ, Sankararajah D, Stewart M et al. Self-expanding metallic stents in the treatment of benign colorectal disease: indications and outcomes. Colorectal Dis 2006; 8: $102-111$

\section{Bibliography}

DOI http://dx.doi.org/

10.1055/s-0032-1326249

Endoscopy 2013; 45: E95-E96

(c) Georg Thieme Verlag KG

Stuttgart · New York

ISSN 0013-726X
Corresponding author

\section{R. Di Mitri, MD}

Via Strada Ferrata 44/D

90046, Monreale

Palermo

Italy

Fax: +39-091-6663053

robdimitri68@yahoo.it 